\title{
An Autonomous Molecular Transport System Using DNAs and Motor Proteins in Molecular Communication
}

\author{
S. Hiyama ${ }^{1,2}$ and $Y$. Moritani ${ }^{1}$ \\ ${ }^{1}$ Research Laboratories, \\ NTT DoCoMo, Inc. \\ 3-5 Hikarinooka, Kanagawa \\ 239-8536, Japan \\ $+81-46-840-3811$ \\ hiyama@nttdocomo.co.jp \\ T. Suda ${ }^{1,3}$ \\ ${ }^{3}$ Information and Computer Science, \\ University of California, Irvine \\ Irvine, CA \\ 92697-3425, USA \\ $+1-949-824-5474$ \\ suda@ics.uci.edu
}

\author{
T. Shima ${ }^{2}$ and K. Sutoh ${ }^{2}$ \\ ${ }^{2}$ Department of Life Sciences, \\ The University of Tokyo \\ 3-8-1 Komaba, Tokyo \\ 153-8902, Japan \\ +81-3-5454-6769 \\ sutoh@bio.c.u-tokyo.ac.jp
}

\begin{abstract}
This paper describes a molecular transport system in molecular communication that uses the machinery in living cells. The molecular transport system requires: 1) loading of the specified cargo molecules at a loading site (at a sender); 2) transport of the loaded cargoes to an unloading site (to a receiver); and 3) unloading of the transported cargoes at the unloading site, all without using external stimuli. Through the DNA strand exchange at a loading site and at an unloading site, and through motility of a biological motor system (kinesins and microtubules), the authors of this paper constructed a molecular transport system and demonstrated that kinesin-driven microtubules autonomously load, transport and unload cargoes to which a specified DNA strand is attached.
\end{abstract}

\section{Keywords}

Molecular communication, Molecular transport system, DNA, Motor protein, Autonomous operation

\section{INTRODUCTION}

Molecular communication uses molecules (i.e., chemical signals) as an information medium and allows biological and artificiallycreated nano- or cell-scale devices to communicate over a short distance [1]. It is a new communication paradigm and is different from the existing communication paradigm that uses electromagnetic waves (i.e., electronic and optical signals) as an information medium. In the existing communication, a sender encodes digital information (such as voice, text, and video) onto electromagnetic waves and transmits them to a receiver. The receiver receives the electromagnetic waves and interprets the encoded information. On the other hand, in the molecular communication, a sender encodes biochemical information (such as phenomena and chemical status) onto molecules and transmits them to a receiver. The receiver receives the information encoded molecules and biochemically reacts to the received molecules

Permission to make digital or hard copies of all or part of this work for personal or classroom use is granted without fee provided that copies are not made or distributed for profit or commercial advantage and that copies bear this notice and the full citation on the first page. To copy otherwise, or republish, to post on servers or to redistribute to lists, requires prior specific permission and/or a fee.

Bionetics'07, December 10-13, 2007, Budapest, Hungary

Copyright 2007 ICST 978-963-9799-11-0 (this biochemical reaction represents decoding of the information). The communication speed of molecular communication is slower than that of existing communication, and molecular communication is a stochastic communication. However, molecular communication may carry the information that is not feasible to carry with existing communication (such as the biochemical status of a living organism) between the entities that the existing communication does not apply (such as biological entities). Molecular communication has unique features that are not seen in the existing communication and is not competitive but complementary to the existing communication.

Key research challenges in molecular communication include 1) design of a sender that generates and emits molecules, 2) design of a molecular transport system [2] that directionally transports the emitted molecules from a sender to a receiver, 3) design of a receiver [3] that receives the transported molecules and biochemically reacts to the received molecules, 4) design of a communication interface [4] between a sender and a transport system and also between a transport system and a receiver to allow a generic transport of molecules independent of their characteristics. Molecular communication is inspired by the biological communication mechanisms (e.g., cell-cell communication using hormones) and artificially creates a controllable communication system. This paper focuses on a molecular transport system that uses the machinery in living cells.

In living cells such as eukaryotic cells, biological motors (e.g., kinesins) load/unload particular types of cargo molecules (e.g., vesicles) without using external stimuli and transport them along cytoskeletal filaments (e.g., microtubules (MTs)) using the energy of adenosine triphosphate (ATP) hydrolysis (in vivo) [5]. Because of these biological capabilities of autonomous loading/unloading and transport of specified cargoes that biological motors have, there is considerable interest in incorporating kinesins and MTs into artificially-created (in vitro) transporters and actuators in nano- or cell-scale systems and applications [2], [6]-[11].

Since a reverse geometry of MT motility on kinesins (in vitro gliding assay) was successfully demonstrated [12], reconstituted MT motility has been receiving increasing attention. Using MT motility in a molecular transport system allows loading of specified cargoes onto gliding MTs at a given loading site (at a given sender), directionally transporting the loaded cargoes toward a given unloading site (to a given receiver), and then 
a

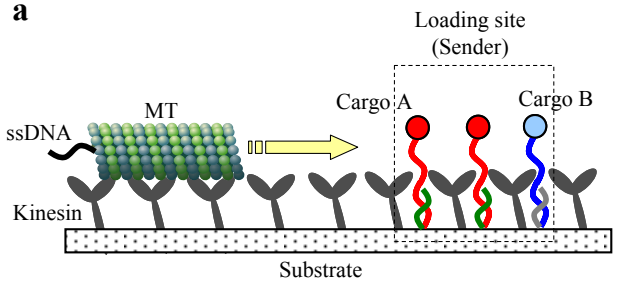

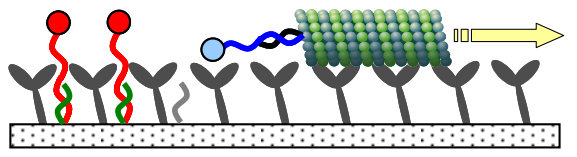

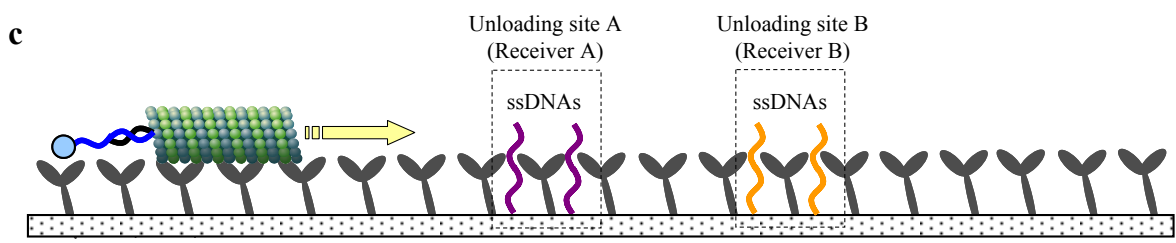

d

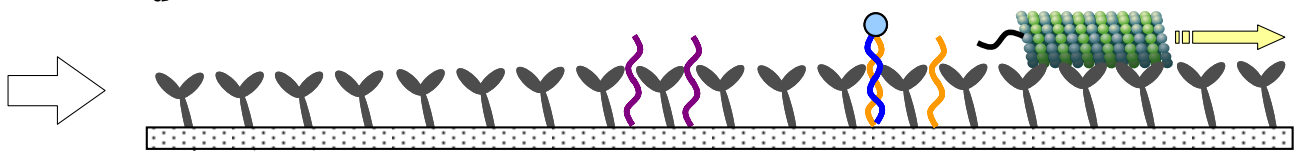

Figure 1. Schematic diagram of a proposed molecular transport system.

unloading the transported cargoes from the MTs at the unloading site, all without using external stimuli. Such autonomous loading/unloading of and directional transport of specified cargoes may help create highly miniaturized on-chip-systems such as molecular sorters and molecular sensors as well as molecular communication systems.

In achieving directional transport of cargoes in a molecular transport system, existing techniques using preconfigured microlithographic tracks [6]-[7] may apply to control the transport direction of cargoes loaded onto gliding MTs. However, in achieving autonomous loading/unloading of cargoes, a new approach is required because existing systems require external stimuli such as UV-light exposure [8]-[9], ligand supplementation [10], restriction enzyme digestion or temperature fluctuation [11] to unload cargoes from gliding MTs; thus making those systems non-autonomous.

This paper describes design and empirically study of a molecular transport system that autonomously loads/unloads specified cargoes using DNA strand exchange and that transports the loaded cargoes using the reverse geometry of MT motility on kinesins. Our empirical results show that kinesin-driven MTs autonomously load and unload, as well as transport, cargoes specified by particular base sequences of DNA.

\section{SYSTEM DESIGN}

The molecular transport system described in this paper achieves autonomous loading/unloading of specified cargoes using DNA strand exchange and transport of the loaded cargoes using the reverse geometry of MT motility on kinesins (Figure 1). In order to use the DNA hybridization and strand exchange, each gliding
MT, cargo, loading site (sender) and unloading site (receiver) is labeled with a single-stranded DNA (ssDNA). Note that the length of an ssDNA attached to an MT is designed to be longer than that of the loading site, and is also designed to be shorter than that of the cargo. Note that the length of an ssDNA attached to a cargo is designed to be as long as that of the unloading site.

Each cargo is weakly trapped at a loading site through DNA hybridization between an ssDNA attached to the cargo (a red or a blue strand) and an ssDNA immobilized onto a substrate (a green or a gray strand) (Figure 1a). The ssDNA for the cargo is designed to be either complementary (a blue strand) or noncomplementary (red strands) to that of the MT (a black strand). When an MT labeled with an ssDNA passes through a given loading site (a given sender), a cargo labeled with an ssDNA complementary to that of the MT (cargo B) is selectively loaded onto the gliding MT through DNA strand exchange without using external stimuli (Figure 1b), while cargoes labeled with a noncomplementary ssDNA remain at the loading site. This loading process through DNA strand exchange may be initiated by the energy state transition from an unstable and high-energy state (i.e., DNA hybridization between ssDNAs attached to the loading site and to the cargo) to a stable and low-energy state (i.e., DNA hybridization between ssDNAs attached to the MT and to the cargo) that takes place naturally.

The cargo loaded onto the MT (i.e., an MT-cargo complex) is transported by MT motility on kinesins toward given unloading sites (given receivers) (Figure 1c).

To achieve autonomous unloading at a given unloading site, the ssDNA attached to each unloading site is designed to be either 

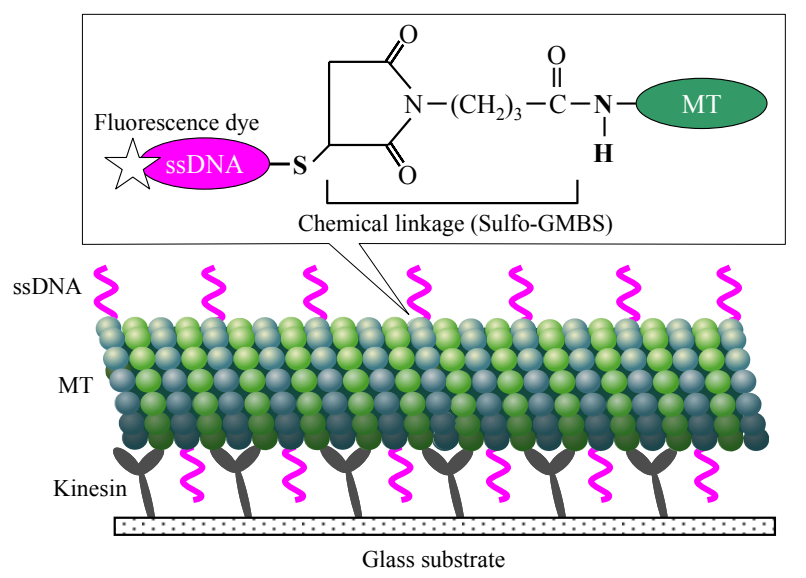

Figure 2. Schematic diagram of an ssDNA-labeled MT and configuration of the in vitro gliding assay.

complementary (orange strands) or non-complementary (purple strands) to that attached to the cargo. When the MT-cargo complex passes through an unloading site, the cargo labeled with an ssDNA complementary to that attached to the unloading site (unloading site B) is selectively unloaded from the gliding MT through DNA strand exchange without using external stimuli (Figure 1d). This unloading process through DNA strand exchange may be initiated by the energy state transition from an unstable and high-energy state (i.e., DNA hybridization between ssDNAs attached to the MT and to the cargo) to a stable and lowenergy state (i.e., DNA hybridization between ssDNAs attached to the unloading site and to the cargo) that takes place naturally. Note that ssDNA-labeled MTs which unloaded cargoes continue to glide over immobilized kinesins and may load new cargoes.

\section{EXPERIMENTAL RESULTS}

To construct an autonomous molecular transport system shown in Figure 1, the authors of this paper first examined various chemical linkages to label MTs with ssDNAs and found that Sulfo-GMBS that cross-links thiolated ssDNAs and amino groups of MTs, as schematically shown in Figure 2, was suitable for dense labeling of MTs with ssDNAs (to maximize the probability of successfully loading of a cargo), while maintaining smooth gliding of labeled MTs on kinesins. The in vitro gliding assay for densely labeled MTs and for unlabeled MTs showed that the densely labeled MTs glided smoothly without pausing or detaching from a surface covered with kinesins, although their average gliding speed $(0.34$ $\mu \mathrm{m} / \mathrm{s})$ decreased by half compared to that of unlabeled MTs $(0.66$ $\mu \mathrm{m} / \mathrm{s})$.

Cargoes (polystyrene microbeads with $1 \mu$ m-diameter) were weakly trapped at a loading site through DNA hybridization between 23-mer ssDNAs (labeled with fluorescence dyes) attached to the cargo and 7-mer ssDNAs immobilized onto a glass substrate. It was observed through fluorescence microscopy that the trapped cargoes were selectively loaded onto gliding MTs labeled with complementary 15 -mer ssDNAs, and the resulting MT-cargo complexes glided on the surface covered with kinesins and transported the loaded cargoes (Figure 3). A very limited

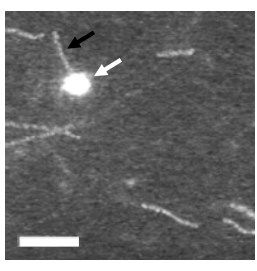

$0 \mathrm{sec}$

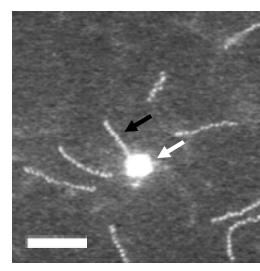

$16 \mathrm{sec}$
Figure 3. Time-lapsed fluorescence images of cargo transport by a single MT. The black and white arrows point to the gliding MT and the cargo-bead, respectively. The scale bars correspond to $3 \mu \mathrm{m}$.

number of cargoes labeled with non-complementary ssDNAs were loaded onto the gliding MTs, confirming that loading of cargoes onto gliding MTs is done through the DNA strand exchange.

It was also observed through fluorescence microscopy that loaded cargoes were selectively unloaded from the gliding MTs at an unloading site where complementary 23-mer ssDNAs with high concentration were immobilized onto a glass substrate, while a very limited number of cargoes were unloaded from gliding MTs at an unloading site of non-complementary 23-mer ssDNAs. These results indicate that unloading of cargoes from the gliding MT is done through the DNA strand exchange.

\section{CONCLUSIONS}

This paper describes a design and the first implementation of autonomous loading/unloading of and transport of specified cargo molecules using the DNA strand exchange and the MT motility on kinesins. The authors of this paper demonstrated that kinesindriven MTs autonomously load, transport and unload cargoes to which a specified DNA strand was attached. Such autonomous and selective loading/transport/unloading mechanisms may help create highly miniaturized on-chip bio-hybrid communication systems that transport information encoded molecules from a sender to a receiver. Incorporating the molecular transport system into a microfluidic channel will be the focus of our next experiments.

\section{ACKNOWLEDGMENTS}

The authors of this paper thank Prof. A. Suyama and Dr. M. Takinoue of the Department of Life Sciences and the Institute of Physics, The University of Tokyo for designing the ssDNA base sequences. The authors also thank Prof. Y. Y. Toyoshima and Mr. K. Furuta of the Department of Life Sciences, The University of Tokyo for providing kinesin-expressing plasmids. As well, the authors thank Dr. T. Inoue of the Institute of Medical Science, The University of Tokyo and Dr. Y. Isogawa of the Effector Cell Institute, Inc., for their help with the biochemical experiments conducted in this paper.

\section{REFERENCES}

[1] Hiyama, S., Moritani, Y., Suda, T., Egashira, R., Enomoto, A., Moore, M., and Nakano, T. Molecular communication. In 
Proceedings of the NSTI Nanotechnology Conference and Trade Show (Nanotech'05), 3, (Anaheim, USA, May 2005), 391-394.

[2] Hiyama, S., Isogawa, Y., Suda, T., Moritani, Y., and Sutoh, $\mathrm{K}$. A design of an autonomous molecule loading/transporting/unloading system using DNA hybridization and biomolecular linear motors. In Proceedings of the European Nano Systems (ENS'05), (Paris, France, Dec. 2005), 75-80.

[3] Sasaki, Y., Hashizume, M., Maruo, K., Yamasaki, N., Kikuchi, J., Moritani, Y. Hiyama, S., and Suda, T. Controlled propagation in molecular communication using tagged liposome containers. In Proceedings of the First International Conference on Bio Inspired Models of Network, Information and Computing Systems (BIONETICS'06), (Cavalese, Italy, Dec. 2006).

[4] Moritani, Y., Nomura, S. M., Hiyama, S., Akiyoshi, K., and Suda, T. A molecular communication interface using liposomes with gap junction proteins. In Proceedings of the First International Conference on Bio Inspired Models of Network, Information and Computing Systems (BIONETICS'06), (Cavalese, Italy, Dec. 2006).

[5] Vale, R. D. The molecular motor toolbox for intracellular transport. Cell, 112, (Feb. 2003), 467-480.

[6] Hiratsuka, Y., Tada T., Oiwa, K., Kanayama, T., and Uyeda, T. Q. P. Controlling the direction of kinesin-driven microtubule movements along microlithographic tracks. Biophys. $J ., 81$, (Sep. 2001), 1555-1561.
[7] Hess, H., Matzke, C. M., Doot, R. K., Clemmens, J., Bachand, G. D., Bunker, B. C., and Vogel, V. Molecular shuttles operating undercover: a new photolithographic approach for the fabrication of structured surfaces supporting directed motility. Nano Lett., 3, (Dec. 2003), 1651-1655.

[8] Kato, K., Goto R., Katoh, K, and Shibakami, M. Microtubule-cyclodextrin conjugate: functionalization of motile filament with molecular inclusion ability. Biosci. Biotechnol. Biochem., 69, (Mar. 2005), 646-648.

[9] Weibel, D. B., Garstecki, P., Ryan, D., Diluzio, W. R., Mayer, M., Seto, J. E., and Whitesides, G. M. Microoxen: microorganisms to move microscale loads. Proc. Natl. Acad. Sci. U.S.A., 102, (Aug. 2005), 11963-11967.

[10] Hirabayashi, M., Taira, S., Kobayashi, S., Konishi, K., Katoh, K., Hiratsuka, Y., Kodaka, M., Uyeda, T. Q. P., Yumoto, N., and Kubo, T. Malachite green-conjugated microtubules as mobile bioprobes selective for malachite green aptamers with capturing/releasing ability. Biotechnol. Bioeng., 94, (June 2006), 473-480.

[11] Taira, S., Du, Y.-Z., Hiratsuka, Y., Konishi, K., Kubo, T., Uyeda, T. Q. P., Yumoto, N., and Kodaka, M. Selective detection and transport of fully matched DNA by DNAloaded microtubule and kinesin motor protein. Biotechnol. Bioeng., 95, (Oct. 2006), 533-538.

[12] Howard, J., Hudspeth, A. J., and Vale, R. D. Movement of microtubules by single kinesin molecules. Nature, 342, (Nov. 1989), 154-158. 